\title{
Mobile Liquid 2D Scatter Space (ML2DSS)
}

A visual interactive information space (ispace) for displaying large amounts of information and allowing simple vision-based knowledge discovery on small screen sizes

Carsten Waldeck, Dirk Balfanz

ZGDV - Computer Graphics Center, Dept. Z3: Mobile Information Visualization and infoverse.org

\section{Executive Summary}

This (video-) paper describes a user interface concept which facilitates browsing of comparably large amounts of information on small screens.

The viewing concept is based on a visually optimized star field display ([1] Ahlberg \& Shneiderman 1994) and is introducing new concepts like liquid browsing (an expansion lens with pressure controlled magnetic force simulation), selection based filtering and representation manipulation, multimotion behavior tagging and continuous state animation. All this creates a very versatile information space with a liquid-like look and feel, which can be used in a very intuitive way, providing all the advantages of a mature interactive scatter plot and can easily be scaled to different screen sizes without any adjustment efforts.

This paper focuses on visual design and interaction details and emphasizes the importance of the visua interactive quality for mobile information visualization.

\section{Introduction and Problem Description}

In Mobile Information Visualization, one of the big issues is the problem of small and a multitude of different screen sizes. In spite of diverse screen concepts like roll up displays, or digital paper, it is most likely that we will continue to battle with the limited screen spaces of handheld computers for quite some time. Further, the related issue of constantly having to adapt the view to different screen sizes presents a complex problem.

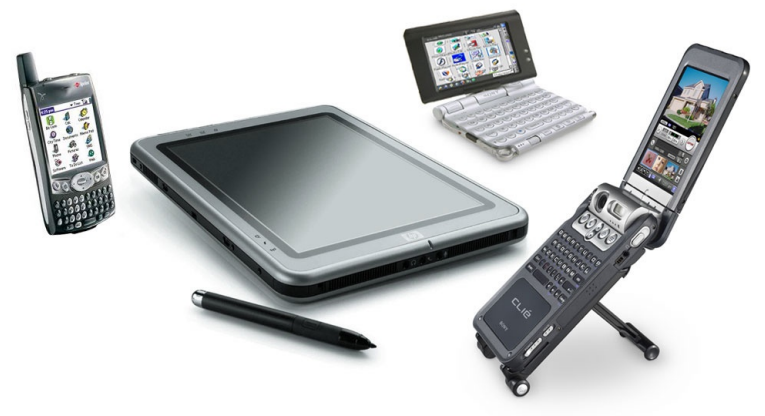


A common table view on a Pocket PC or PDA allows us to only look at 10-20 objects at a time without scrolling, allowing to sort and compare according to one single criterion. An interactive 2D scatter plot approach would allow much larger amounts of information to be visualized and is simultaneously sortable based on two criteria. In addition, comparisons based on a multitude of criteria are possible at the same time (according to mapping and expressivity of the visual parameters: size opacity, color value, shape, orientation, ... or time animation of all these parameters, which results in visual representations like motion, growing/shrinking, pulsing, rotation, shape morphing,...).

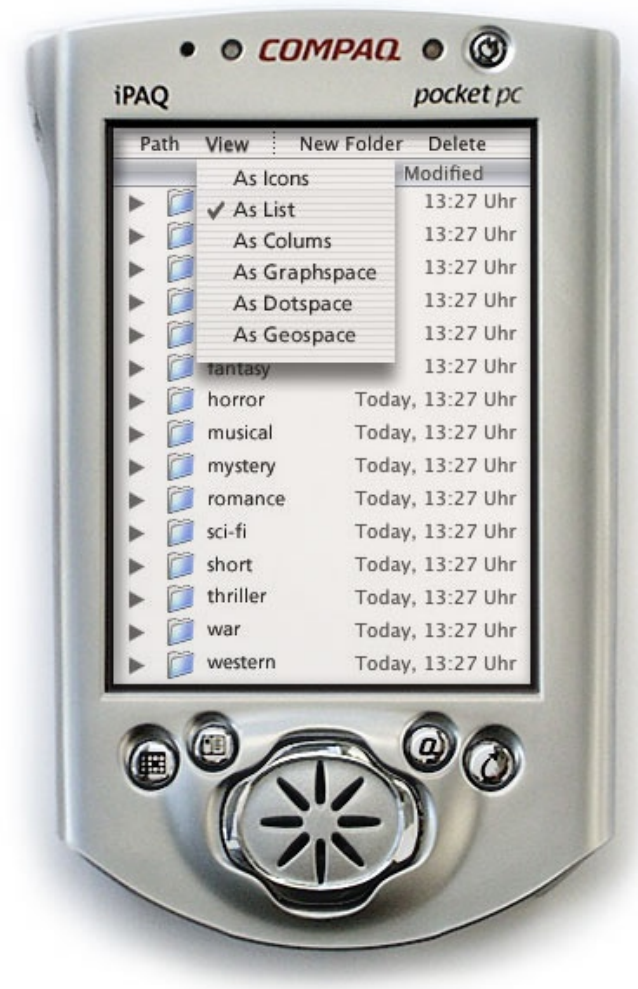

One disadvantage of a 2D scatter plot approach however, is the possibility of the information objects to overlap to some extent hindering readability and perceptibility of the information or, worst case, even making it impossible. To solve this problem, zoom and lens functions have been implemented ([5] Sarkar \& Brown 1992), which allow the (partial) enlargement of the information to counter this problem. Still, browsing high information densities this way proved to be a very

challenging task, because a simple enlargement based fisheye lens does not reduce the overlapping and very often has to deal with heavy distortion issues. 


\section{Solutions}

2.1. Liquid browsing: To address the above-mentioned problems, we are proposing a distance manipulation based expansion lens (in contrast to the common size manipulation based magnification lens). By not enlarging the information objects themselves, but rather the spaces between them, we are solving our real problem - the overlapping - much more effectively.

By using this kind of "lens" model, we are achieving much greater clarity with less space consumption and minimal context distortion.
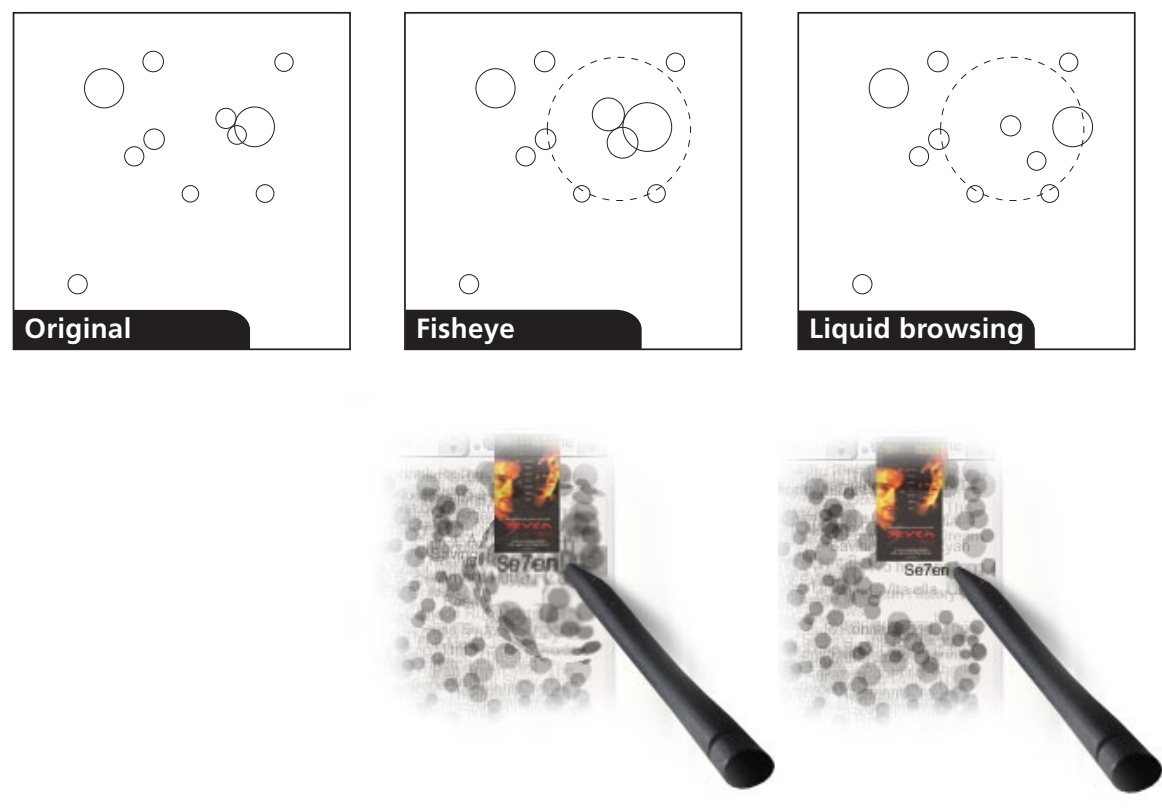

Comparison on screen: enlargement based lens (fisheye) with distortion on left vs. distance based lens (liquid browsing) on right
In addition, we are using rollover opacity raising for the typography to separate the related text tag from the background. Supplementary, the offset motion of the lens increases this intention.
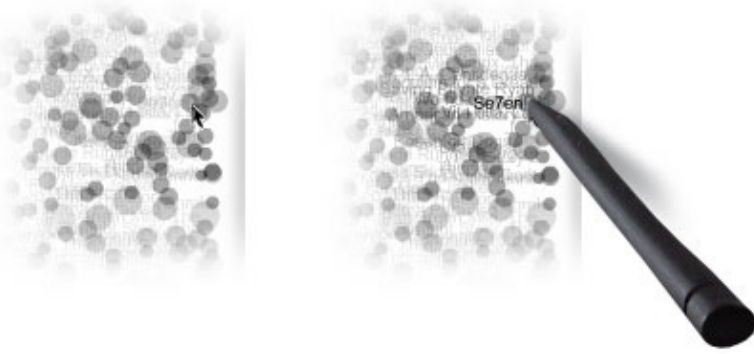

Comparison: Original view on left vs. opacity raised typo-detail (to better understand this effect please refer to video).

By using the pressure the user puts on the screen with the input device (naturally a pen) to control strength and radius of the expansion lens (also "push lens") and adding oil- or liquid-like friction and acceleration forces, we achieved a very direct 1:1 "impression", which makes it intuitively controllable and creates a nicely flowing browsing experience. This is why people liked to refer to it as "liquid browsing".

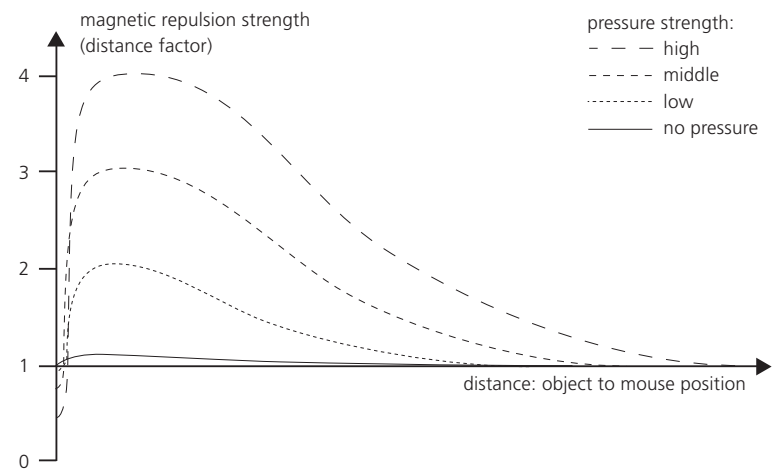




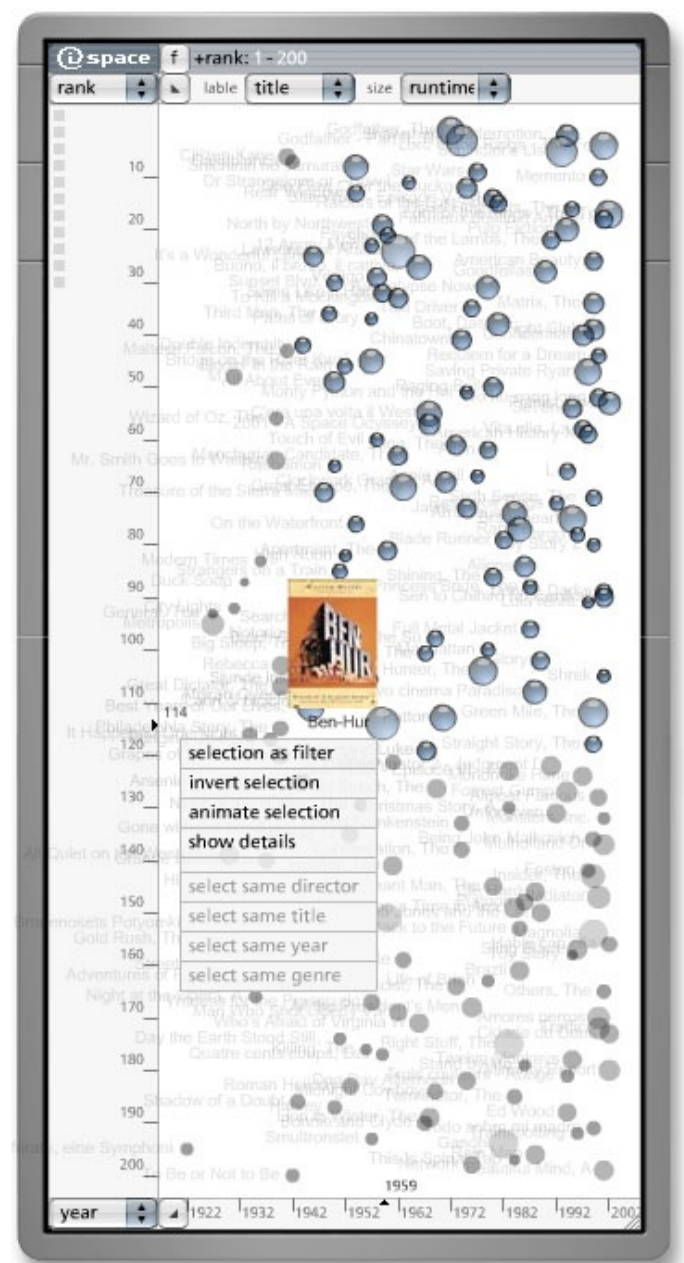

4 2.2. Selection based manipulation: To reduce input effort (big issue on mobile devices without a proper keyboard) and to increase the effective display space, we propose selection based filtering and selection based representation adjustment in addition to (or instead of) sliders or text input for adjusting the filters and directly manipulating the views:

Simply draw a selection and choose an action from the context menu to set the axis filters, highlight relations or undertake other representation manipulations.

One very important issue is finding the right values for the visual variables (like size, opacity, color value, shape, orientation,...) to achieve the maximal coherence and expressiveness of the visual representation ([4] Bertin, 1983). To examine this subject, we built a prototype which allowed us to freely set every single one of those correlations and compare the expressivity and the perceptibility of a combination of several visual parameters used simultaneously.

Naturally, it is advantageous to avoid contrary correlations of visual parameters to achieve the maximal expressiveness. But for our prototype, we used this effect on purpose to further increase the information density: by using a contrary coding of size and opacity, it was possible to reduce the overlapping problem even more and increase visual clarity (bigger objects are less opaque).
2.3. Multimotion behavior tagging: Motion or animation like blinking or flashing have often been used for visualization purposes. Many of our studies have revealed that it is not only possible to easily perceive macro- and micro-movements at the same time, but also multiple syncronized micro-movements if they are used in succession.

When implementing our prototype, we used this effect to express the position of the information object by means of motion, which allowed us to visualize two more dimensions without loss of visual clarity (see movie). Another very good way of adding more simultaneously visualizable dimensions is the synchronization of additional views. However, this possibility is limited due to little available screen space on mobile devices.
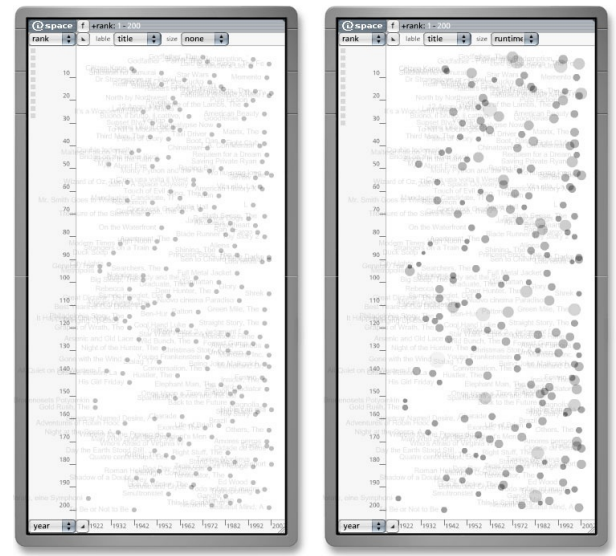

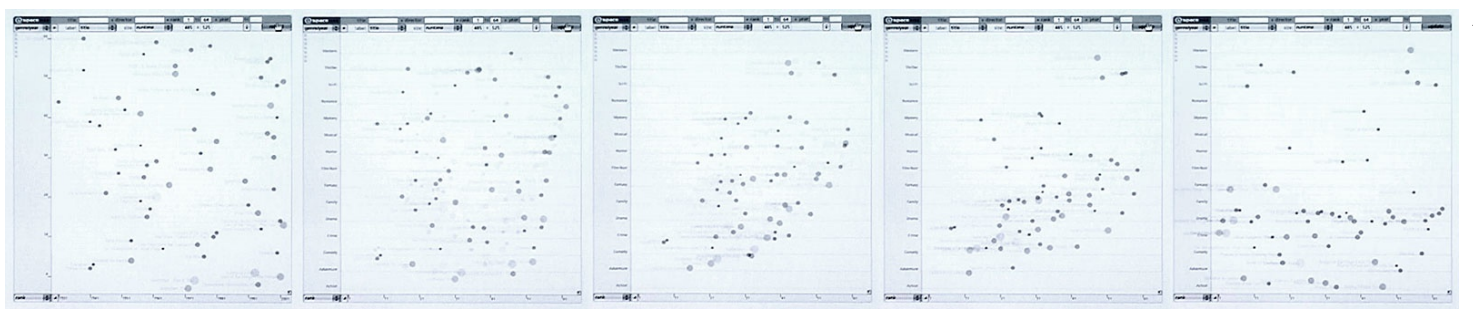

2.4. Continuous state animation: All position changes of information objects are continuously animated, which explains complex processes in a very straightforward way and makes it possible to detect clusters of object masses and very detailed object movements simultaneously. To better understand this effect please see the video. 


\section{Prototype}

The first Mobile Liquid Scatter Space prototypes were implemented in Macromedia Flash, Java and Objectivec (macOSX). Most of what you can see in the movie is based on a Flash application that reads an XML database of the 250 most popular movies of the world [7]

You can find this movie at:

http://www.infoverse.org/ispace/ispace_movie.htm

A prototype can also be experienced at:

http://www.infoverse.org/ispace/ispace_flash.htm

The Mobile Liquid 2D Scatter Space version you see in the movie is optimized for fast devices with pressuresensitive screens (something like a tablet PC today). We also have a version for HP ipaq (Flash), but it is very slow and pressure sensitivity and rollover functionality are missing. We believe there will be small, fast and pressuresensitive handhelds in the future and want to focus on those kinds of devices. Most of the prototypes are tested and presented on a fast computer with a Wacom Cintiq (Interactive Pen Display).

\section{Conclusion and Future Work}

This paper has introduced liquid browsing as an interaction method to solve the overlapping problem of interactive 2D scatter plots. Optimizing visual interactive details is crucial, especially for the mobile domain. Though empirical studies still have to be done, we believe that ML2DSS can be very useful as a versatile and very flexible ispace in a wide range of applications (file systems, media libraries, map browsing, email,...)

ML2DSS is a part of the "iworld" project, which is about a versatile multiple view information browser with a special focus on semantic web browsing.

\section{References}

[1] Ahlberg, Christopher and Shneiderman, Ben (1994): Visual Information Seeking: Tight Coupling of Dynamic Query Filters with Star field Displays. Proc. of CHI 1994. ACM, New York, pp 313-317.

[2] Ahlberg, Christopher and Shneiderman, Ben (1994): The AlphaSlider: A Compact and Rapid Selector. Proc. of CHI 1994. ACM, New York, pp 365-372.

[3] Bederson, Benjamin and Hollan, James (1994): Pad++: A Zooming Graphical Interface for Exploring Alternate Interface Physics. Proc. of UIST 1994, ACM, New York.

[4] Bertin, Jacques. Semiology of Graphics. The University of Wisconsin Press, 1983.

[5] Davidson, Neil and Dunlop, Mark D (2000): Visual information seeking on palmtop devices. Proc. of $\mathrm{HC}$ 2000

[6] Sarkar, Manojit and Brown, Marc. Graphical Fisheye Views of Graphs. Proc. of CHI 1992, ACM, New York, pp 83-91.

[7] Internet Movie Database: http://www.imdb.com

\section{Contact Information}

Carsten Waldeck

ZGDV - Computer Graphics Center

Dept. Z3, MIV (Mobile Information Visualization)

Fraunhofer Str. 5

64283 Darmstadt, Germany

Tel : +49 (0)6151-155-623

http://www.zgdv.de

http://www.infoverse.org

carsten@infoverse.org 